\title{
Elucidating the pathophysiology of left bundle branch block related perfusion defects
}

\author{
Yuka Otaki, MD, PhD, ${ }^{a}$ Robert J. H. Miller, MD, ${ }^{\mathrm{a}, \mathrm{b}}$ and Piotr J. Slomka, PhD ${ }^{\mathrm{a}}$ \\ a Department of Imaging (Division of Nuclear Medicine), Medicine, and Biomedical Sciences, \\ Cedars-Sinai Medical Center, Los Angeles, CA \\ b Department of Cardiac Sciences, University of Calgary, Calgary, AB, Canada
}

Received Jun 2, 2020; accepted Jun 2, 2020

doi: $10.1007 / \mathrm{s} 12350-020-02224-y$

See related article, pp. 2910-2922

Patients with left bundle branch block (LBBB) are well known to have perfusion defects in the anteroseptal wall seen on single photon emission computed tomography (SPECT) myocardial perfusion imaging (MPI) often without having disease in the left anterior descending artery (LAD) (Figure 1). The prevalence of this perfusion abnormality with Tc-99m tracers has been reported to be over $50 \%$ and often occurs with exercise or dobutamine stress. ${ }^{1,2}$ Therefore, the guidelines for SPECT-MPI recommend performing pharmacologic vasodilator stress test for patients with LBBB. ${ }^{3}$ Several mechanisms have been proposed to explain this perfusion abnormality such as decreased blood flow in response to lower septal resting oxygen demand, ${ }^{1,4}$ diastolic compression of septal perforators, ${ }^{5}$ septal microvessel compression secondary to abnormal and delayed septal contraction/relaxation and redistribution of circumferential shortening. ${ }^{1,6}$ However, the relative contributions of these proposed mechanisms are not yet fully understood.

In this issue of the Journal, Hedeer and colleagues ${ }^{7}$ studied 23 patients with both SPECT and cardiovascular magnetic resonance (CMR) imaging available, in order to elucidate the etiology of LBBB-related perfusion defects. The typical LBBB perfusion defect pattern was visually defined as a nonreversible anteroseptal

Reprint requests: Yuka Otaki, MD, PhD, Department of Imaging (Division of Nuclear Medicine), Medicine, and Biomedical Sciences, Cedars-Sinai Medical Center, 8700 Beverly Boulevard, Suite. 1258, Los Angeles, CA 90048; Yuka.Otaki@cshs.org

J Nucl Cardiol 2021;28:2923-6.

$1071-3581 / \$ 34.00$

Copyright (C) 2020 American Society of Nuclear Cardiology. perfusion defect without involvement of the apex. By SPECT-MPI, 13 patients were considered to have a typical LBBB defect pattern and 10 patients were not. The population of patients with LBBB assessed by noninvasive multimodality imaging including SPECT and CMR with cine, strain and late gadolinium enhancement (LGE) studied by Hedeer et al. is unique. This allowed for novel comparisons of myocardial fibrosis, wall motion, myocardial strain, and dyssynchrony between patients with and without typical LBBB perfusion defect patterns.

This study makes a few observations, which provide insight into the etiology of LBBB-related perfusion defects. First, lower myocardial radiotracer counts were present in the septal wall compared to the lateral wall in both groups of patients. Although the authors identified modest differences in wall thickness, it is unlikely to be the underlying mechanism for this finding. Sugihara et al. demonstrated that the lower septal count is more prominent at end-systole compared to end-diastole. ${ }^{8}$ In their study, the septal to lateral wall count ratio was lower in patients with LBBB compared to control patients on nongated images, while it was similar on end-diastolic images. In addition, in patients with LBBB the count increase from end-diastole to end-systole was smaller in the septal region compared to the lateral region. ${ }^{8}$ These findings suggest that the defect may be related to decreased wall thickening in the septal region.

LBBB activation leads to a distinct contraction pattern of wall motion. ${ }^{9}$ CMR imaging allows assessment of septal wall motion abnormality and LV mechanical deformation from cine and tagged images, using principles similar to those utilized in echocardiography, including radial, circumferential or longitudinal strain, timing of myocardial thickening and volume change. ${ }^{10} \mathrm{~A}$ related finding by Hedeer et al. ${ }^{7}$ was reduced radial strain in the septal wall in patients with a typical LBBB perfusion defect pattern compared to 


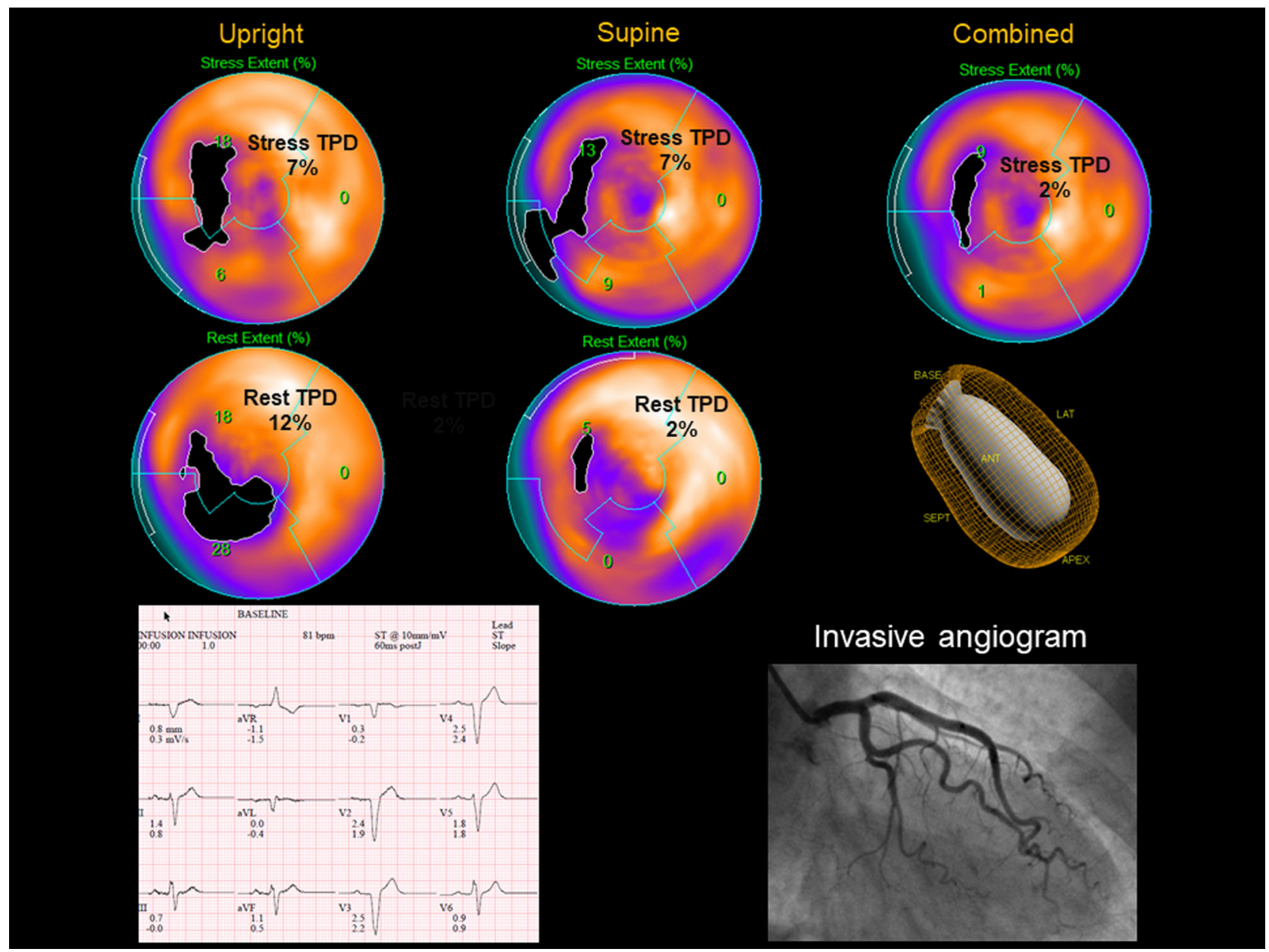

Figure 1. Case example with anteroseptal perfusion defect on regadenoson stress and rest Tc-99m sestamibi D-SPECT-MPI in a 77-year-old female with left bundle branch block. SPECT-MPI showed a perfusion defect in anteroseptal wall at stress and rest. Stress TPD (total perfusion deficit) derived by QPS software (Cedars-Sinai Medicla Center, Los Angeles, CA) ${ }^{17}$ was $7 \%$ on upright and supine positions, and combined stress TPD was $2 \%$. Rest TPD was $12 \%$ on upright position and $2 \%$ on supine position. LV wall motion demonstrated severe hypokinesis in the septal and apical walls. Invasive angiogram performed 2 weeks after the SPECT-MPI showed nonobstructive coronary artery disease.

those without, while there was no difference in the lateral wall between groups. This suggests that the extent and distribution of the perfusion defect in patients with LBBB might correlate with LV deformation. Vernooy et al. also demonstrated decreased septal circumferential shortening in a canine model with LBBB induced by radio frequency ablation. ${ }^{6}$ In addition, they demonstrated that myocardial blood flow decreased after induction of LBBB, suggesting that septal hypoperfusion may be contributory.

Few studies have investigated the prevalence of myocardial fibrosis in the anteroseptal wall in patients with LBBB. In the present study, four patients with a typical LBBB defect had any LGE, including two patients with LGE in the septum. Two patients without a typical LBBB defect had LGE in the lateral wall suggesting that it may have masked a relative septal defect. Grigoratos et al. examined the presence and extent of myocardial fibrosis by CMR in 196 patients with nonischemic cardiomyopathy. LGE was observed more commonly in patients with LBBB $(n=83)$ compared to patients with normal intraventricular conduction $(\mathrm{n}=84)$ and was more often located in the ventricular septum $(P<0.001) .{ }^{11}$ The authors suggested that septal fibrosis on CMR might reflect the underlying anatomic interruption of the proximal His-bundle network and 
identify patients who might benefit from pacing the distal portion of the His-bundle branches. ${ }^{12}$ Mahmod et al. evaluated 55 asymptomatic patients with LBBB and demonstrated up to a third of patients with normal echocardiograms had abnormalities on CMR. ${ }^{13}$ The authors concluded that CMR detects sub-clinical cardiomyopathy in a third of asymptomatic patients with LBBB despite normal echocardiograms and provides contributes clinically relevant information in patients with abnormal echocardiograms. Mahrholdt et al. evaluated LGE by CMR in 19 patients with LBBB, without any CAD on invasive coronary angiography, who had fixed perfusion defects. ${ }^{14}$ In their study, subendocardial or transmural LGE, representing myocardial infraction, was not found in any patient. Compared to the previous study, LGE was observed in the anterior or septal wall in two of 13 patients with typical LBBB perfusion defect and patients with significant CAD were not excluded in the present study. Given the spectrum of findings, the relative contribution of myocardial fibrosis to LBBBrelated perfusion defects is most likely driven substantially by characteristics of the studied patient population.

Although the study by Hedeer et al. ${ }^{7}$ offers some important findings, there are some limitations to consider. The study population was small and had inherent selection bias due to including patients referred for both SPECT and CMR imaging. Although the mean LVEF was $\sim 35 \%$, there was little information available regarding the etiology of cardiomyopathy in most patients. Although a minority of patients had ischemic LGE, invasive coronary angiography results were not available to allow the authors to account for extent of coronary artery disease as a potential confounder. Alternative etiologies for $\mathrm{LBBB}$ to be considered include: chronic myocardial fibrosis related to hypertension, valvular heart disease, cardiomyopathies (such as sarcoidosis), endocarditis, or myocarditis. ${ }^{15}$ Importantly, all of those etiologies could potentially impact LV mechanics, perfusion, and fibrosis. Lastly, the authors could have more thoroughly addressed the role of fibrosis if contemporary CMR sequences, such as T1 mapping or extracellular volume estimates, were available.

Overall, the study by Hedeer et al. ${ }^{7}$ suggests that the typical perfusion abnormality seen on SPECT-MPI is related to differences in wall thickening and myocardial deformation. In this selected population, ischemic myocardial fibrosis was not a major contributor. However, it may still be prudent for clinicians to exclude significant CAD using adjunctive modalities such as CMR or computed tomographic attenuation maps. Recently artificial intelligence technique has been developed to greatly improve the detection of disease or prediction of outcome in medical imaging field. ${ }^{16}$ In the future, artificial intelligence techniques could help clinicians to differentiate typical LBBB perfusion defect pattern automatically from CAD-related perfusion defects, but those algorithms will likely be informed by studies like this.

\section{Disclosure}

Dr. Piotr J. Slomka received royalties for nuclear cardiology software from Cedars Sinai. Drs. Yuka Otaki, and Robert J. H. Miller have nothing to disclose.

\section{References}

1. Alexanderson E, Mannting F, Gomez-Martin D, Fermon S, Meave A. Technetium-99m-Sestamibi SPECT myocardial perfusion imaging in patients with complete left bundle branch block. Arch Med Res 2004;35:150-6.

2. Higgins JP, Williams G, Nagel JS, Higgins JA. Left bundle-branch block artifact on single photon emission computed tomography with technetium Tc 99m (Tc-99m) agents: Mechanisms and a method to decrease false-positive interpretations. Am Heart $\mathrm{J}$ 2006;152:619-26

3. Henzlova MJ, Duvall WL, Einstein AJ, Travin MI, Verberne HJ. ASNC imaging guidelines for SPECT nuclear cardiology procedures: Stress, protocols, and tracers. J Nucl Cardiol 2016;23:60639.

4. Kasai T, Depuey EG, Shah AA. Decreased septal wall thickening in patients with left bundle branch block. J Nucl Cardiol 2004;11:32-7.

5. Skalidis EI, Kochiadakis GE, Koukouraki SI, Parthenakis FI, Karkavitsas NS, Vardas PE. Phasic coronary flow pattern and flow reserve in patients with left bundle branch block and normal coronary arteries. J Am Coll Cardiol 1999;33:1338-46.

6. Vernooy K, Verbeek XA, Peschar M, Crijns HJ, Arts T, Cornelussen RN, et al. Left bundle branch block induces ventricular remodelling and functional septal hypoperfusion. Eur Heart J $2005 ; 26: 91-8$.

7. Hedeer F, Ostenfeld E, Hedén B, Prinzen FW, Arheden H, Carlsson M, et al. To what extent are perfusion defects seen by myocardial perfusion SPECT in patients with left bundle branch block related to myocardial infarction, ECG characteristics, and myocardial wall motion? J Nucl Cardiol 2020. https://doi.org/10. 1007/s12350-020-02180-7.

8. Sugihara H, Tamaki N, Nozawa M, Ohmura T, Inamoto $\mathrm{Y}$, Taniguchi Y, et al. Septal perfusion and wall thickening in patients with left bundle branch block assessed by technetium-99m-sestamibi gated tomography. J Nucl Med 1997;38:545-7.

9. Prinzen FW, Hunter WC, Wyman BT, McVeigh ER. Mapping of regional myocardial strain and work during ventricular pacing: Experimental study using magnetic resonance imaging tagging. J Am Coll Cardiol 1999;33:1735-42.

10. Sohal M, Duckett SG, Zhuang X, Shi W, Ginks M, Shetty A, et al. A prospective evaluation of cardiovascular magnetic resonance measures of dyssynchrony in the prediction of response to cardiac resynchronization therapy. J Cardiovasc Magn Reson 2014;16:58.

11. Grigoratos C, Liga R, Bennati E, Barison A, Todiere G, Aquaro $\mathrm{GD}$, et al. Magnetic resonance imaging correlates of left bundle branch disease in patients with nonischemic cardiomyopathy. Am J Cardiol 2018;121:370-6. 
12. Lewis AJM, Foley P, Whinnett Z, Keene D, Chandrasekaran B. His bundle pacing: A new strategy for physiological ventricular activation. J Am Heart Assoc 2019;8:e010972.

13. Mahmod M, Karamitsos TD, Suttie JJ, Myerson SG, Neubauer S, Francis JM. Prevalence of cardiomyopathy in asymptomatic patients with left bundle branch block referred for cardiovascular magnetic resonance imaging. Int $\mathrm{J}$ Cardiovasc Imaging 2012;28:1133-40.

14. Mahrholdt H, Zhydkov A, Hager S, Meinhardt G, Vogelsberg H, Wagner A, et al. Left ventricular wall motion abnormalities as well as reduced wall thickness can cause false positive results of routine SPECT perfusion imaging for detection of myocardial infarction. Eur Heart J 2005;26:2127-35.
15. Surkova E, Badano LP, Bellu R, Aruta P, Sambugaro F, Romeo G, et al. Left bundle branch block: From cardiac mechanics to clinical and diagnostic challenges. Europace 2017;19:1251-71.

16. Piotr J, Slomka RJM, Ivana I, Damini D. Application and translation of artificial intelligence to cardiovascular imaging in nuclear medicine and noncontrast CT. Semin Nucl Med 2020;0:1-10.

17. Slomka PJ, Nishina H, Berman DS, Akincioglu C, Abidov A, Friedman JD, et al. Automated quantification of myocardial perfusion SPECT using simplified normal limits. J Nucl Cardiol 2005; 12:66-77.

Publisher's Note Springer Nature remains neutral with regard to jurisdictional claims in published maps and institutional affiliations. 